\title{
Acknowledgment to Reviewers of Computation in 2021
}

\author{
Computation Editorial Office
}

Citation: Computation Editorial Office. Acknowledgment to Reviewers of Computation in 2021. Computation 2022, 10, 20. https://doi.org/10.3390/ computation 10020020

Published: 26 January 2022

Publisher's Note: MDPI stays neutral with regard to jurisdictional claims in published maps and institutional affiliations.

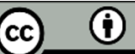

Copyright: $\odot 2022$ by the author. Licensee MDPI, Basel, Switzerland. This article is an open access article distributed under the terms and conditions of the Creative Commons Attribution (CC BY) license (https://creativecommons.org/licenses/by/4.0/).

MDPI AG, St. Alban-Anlage 66, 4052 Basel, Switzerland

Rigorous peer-reviews are the basis of high-quality academic publishing. Thanks to the great efforts of our reviewers, Computation was able to maintain its standards for the high quality of its published papers. Thanks to the contribution of our reviewers, in 2021, the median time to first decision was 15 days and the median time to publication was 38.5 days. The editors would like to extend their gratitude and recognition to the following reviewers for their precious time and dedication, regardless of whether the papers they reviewed were finally published:

Adam, George K.

Ahmadian, Ali

Akrami, Mohammad

Al-Shargie, Fares

Ambrożkiewicz, Bartłomiej

Amić, Ana

Anagnostopoulos, Nikolaos Athanasios

Andrianov, Igor

Angiulli, Giovanni

Antunes Do Carmo, José Simão

Arjunan, Arun

Arteta, Alberto

Artiemjew, Piotr

Arunachalam, Prabhakarn

Ataei, Mehdi

Atuchin, Victor

Aullon, Gabriel

Bach, Mirjana Pejic

Baleanu, Dumitru

Barsi, Dario

Barukčić, Marinko

Batchuluun, Ganbayar

Bayer, Ilker

Beck, Krzysztof

Bergmann, José Ricardo

Bernat, Jakub

Biscarini, Chiara

Blavette, Anne

Brito-Santana, Julio

Briz-Redón, Álvaro

Broadbridge, Philip

Buonocore, Francesco

Cabaleiro, David

Caceres, Máximo Larry López
Caggiano, Antonio

Calore, Enrico

Camposo Pereira, Artur

Cárdenas Alzate, Pedro Pablo

Casella, Girolamo

Casida, Mark

Cavalieri, Francesco

Cerón-Carrasco, José P.

Che Idris, Azam

Chen, Jiawei

Chen, Kuen-Suan

Chen, Nai-Hua

Chesneau, Christophe

Chintalapudi, Nalini

Chumachenko, Dmytro

Chung, Gunhui

Cipu, Elena-Corina

Cotas, Carla

Crivoi, Alexandru

Cuadrado-Calle, David

Custodio, Jean M. F.

D'Acierno, Luca

D'Aniello, Giuseppe

Damaj, Issam W.

Daneshfaraz, Rasoul

Dassios, Ioannis

Dasygenis, Minas

De Brevern, Alexandre G.

Determan, John

Deza, Antoine

Diaconita, Vlad

Díaz García, Antonio F.

Dinis, Maria Alzira Pimenta

Dixon, David 
Dobrițoiu, Maria

Doñate, Cristina Martín

Douvi, Eleni

Dressel, Andrew

Drewniak, Józef

Dulf, Eva H.

Dumitru, Cristian-Dragoş

Eftimie, Raluca

Eglitis, Roberts

Elbattah, Mahmoud

Eliwa, Mohammed

Elmogy, Mohammed

Eshghinejadfard, Amir

Esteghamatian, Amir

Falcón Ganfornina, Raúl Manuel

Fantazzini, Dean

Feng, Jinyong

Fernandes, Marcelo

Fernández-Lázaro, Fernando

Ferrara, Massimiliano

Ferreira, Maria

Fidan, Ismail

Fisicaro, Emilia

Flah, Aymen

Floris, Franca

Forero, Jorge Duarte

Fort, Hugo

Foti, Daniel

Fuenmayor, Carlos Alberto

Futschik, Matthias

Galazka, Miroslaw

Galić, Irena

Garai, Massimo

Garcia Navarro, Pigar

Gazan, Rich

Generowicz, Agnieszka

Georgieva, Atanaska

Ghaffar, Adnan

Gligor, Adrian

Gomes, Rahul

González, Francisco Martínez

González, Jose

González-Longatt, Francisco

Gonzalez-Parra, Gilberto

Goraus, Jerzy

Gorb, Leonid G.

Gornicki, Krzysztof

Gosti, Giorgio

Grabski, Jakub Krzysztof

Grega, Robert

Grindeanu, Iulian
Gubar, Elena

Hammouch, Zakia

Hand, Emily

Herrera Corral, Gerardo A.

Heydari, Azim

Holien, Jessica

Houssein, Essam H.

Huang, Jih-Jeng

Hur, Su-Mi

Hussien, Mostafa A.

Iannario, Maria

Ilieva, Galina

Isgrò, Francesco

Ismail, Mohammed

Jackson, Mark James

Jackson, Nathan

Jagtap, Ameya

Jamro, Ernest

Jeong, Young-Seob

Jian, Muwei

Kanbur, Barış Burak

Kang, Dong Gu

Kangalgil, Figen

Karim, Samsul Ariffin Abdul

Karimov, Artur

Karmakar, Nemai Chandra

Karoń, Grzegorz

Kazakov, Aleksandr Leonidovich

Khalid, Hattaf

Khalifa, Amal

Khan, Abdul Samad

Khan, Muhammad Altaf

Khodadadian, Amirreza

Kim, Seok-Kyoon

Kiseleva, Irina

Kizek, Ján

Klement, Peter

Knypiński, Łukasz

Köstler, Harald

Kotowski, Andrzej

Kozlova, Svetlana G.

Krishnasamy, Gopinath

Kumar, Akshay

Kwilinski, Aleksy

Lagaris, Isaac

Lavrentjev, Jüri

Leftheriotis, Georgios

Leijon, Arne

Lesiuk, Grzegorz

$\mathrm{Li}$, Xiaowei

Liao, Zhixue 
Ligęza, Paweł

Liguori, Francesco

Lim, Jae Hyuk

Limiti, Ernesto

Liu, Pengfei

LIU, Shuhong

Longatte-Lacazedieu, Elisabeth

Loureiro, Felipe Dos Santos

Lu, Diannan

$\mathrm{Lu}, \mathrm{Lu}$

Luliński, Piotr

Marcel, Christian Pablo

Marcinkowski, Leszek

Marsano, Davide

Marshall, Jeffrey

Martinez Prata, Diego

Martinez Ramos, Jose Luis

Martínez-Gómez, Pedro

Martins, Jorge

Martins, Jorge N. R.

Marzi, Claudia

Mehrizi, Abbasali Abouei

Mercorelli, Paolo

Merker, Jochen

Mikalef, Patrick

Mironescu, Ion

Mittas, Nikolaos

Mohamed, Mohamed Arif Bin

Moraca, Federica

Mora-Camino, F.

Morel, Benoit

Mortensen, Jennifer C

Moškon, Miha

Mostowska, Joanna

Mousavi, Mohsen

Murea, Cornel Marius

Nagy, Agnes

Nandasena, N. A. K.

Narayan, Darren A.

Nauroze, Syed Abdullah

Nazarovets, Serhii

Németh, Zsolt István

Neumann, Philipp

Neytcheva, Maya G.

Nitulescu, Mihai

Noeiaghdam, Samad

Oliver, Ozohanics

Orosz, Tamás

Orphanou, Kalia

Ouali, Abdelkader

Palacio, José Carlos Escobar
Panholzer, Martin

Papadakis, George

Pasha, Junayed

Patašius, Martynas

Patra, Braja Gopal

Pavelka, Michal

Penchel, Rafael Abrantes

Penenko, Alexey

Pereira, Paulo Rogerio

Pérez-Villanueva, Jaime

Petravić, Janka

Petroșanu, Dana-Mihaela

Pijarski, Pawel

Piltan, Farzin

Pinto, Milena Faria

Pinto, Mónica

Pittino, Federico

Piwowarski, Marian

Politano, Grazia Giuseppina

Postacchini, Matteo

Postnikov, Eugene

Pruncu, Catalin

Pugliese, Luigi

Puška, Adis

Quessy, Jean-François

Raich, Lluís

Rana, Pratip

Rau, Henrike

Ravber, Miha

Ravina-Ripoll, Rafael

Rees, Andrew

Reis, Gregory

Reviznikov, Dmitry L.

Rezig, Sadok

Richtera, Lukáš

Rivera, Gilberto

Rizzo, Emanuele

Robitzsch, Alexander

Rodríguez Mayorga, Mauricio

Roekaerts, Dirk J. E. M.

Roy, Dipankar

Rubio, Jose De Jesus

Ruzzene, Maria

Rychkov, Denis

Sachenko, Anatoli

Sadyrbaev, Felix

Safaei, Mohammad Reza

Sakhnevych, Aleksandr

Salimi, Mehdi

Salvadori, Simone

Sankaranarayanan, Nehru Viji 
Savoldi, Laura

Sawik, Bartosz

Sayed, Khairy

Semlitsch, Bernhard

Setoodeh, AliReza

Shahid, Arsalan

Shamsuzzaman, Mohammad

Shao, Ke

Shin, Donghyuk

Shorstkii, Ivan

Siami-Namini, Sima

Singh, Avinash

Singh, Shiwani

Singla, Rajeev

Sivasubramanian, Jayahar

Smyk, Emil

Song, Hanjung

Sonnino, Giorgio

Sousa, Sérgio F.

Suhartono, Derwin

Swaminathan, N.

Szultka-Młyńska, Małgorzata

Tankiewicz, Matylda

Tant, Katherine M. M.

Tantardini, Christian

Tavelli, Maurizio

Thompson, John D.

Tihon, Denis
Todorov, Michail

Torcicollo, Isabella

Tran, Quoc-Anh

Turan, Cigdem

Van Bommel, Christopher Martin

Vegas Hernández, Jesús M.

Venkatraman, Sitalakshmi

Versaci, Mario

Vieru, Dumitru

Vinayagam, Arangarajan

Vitázek, Ivan

Vizureanu, Petrică

Vrabel, Robert

Wake, Graeme

Wang, Ke

Weichbroth, Paweł

Wilburn, Kathleen M.

Wilkosz, Kazimierz

Witte, Francesco

Xie, Shengkun

Yang, Huaiwen

Yavuz, Mehmet

Yegin, Korkut

Young, Derek

Zafar, Azhar

Zahariev, Federico

Zambrano Martinez, Jorge Luis

Zhou, Jianguo 